\title{
The Characteristics of Moral Judgment of Psychopaths: The Mediating Effect of the Deontological Tendency
}

This article was published in the following Dove Press journal: Psychology Research and Behavior Management

\author{
Shenglan $\mathrm{Li}^{1,2}$ \\ Daoqun Ding ${ }^{1,2}$ \\ Ji Lai ${ }^{1}$ \\ Xiangyi Zhang ${ }^{1,2}$ \\ Zhihui $\mathrm{Wu}^{\prime}$ \\ Chang $\mathrm{Liu}^{3}$ \\ 'Department of Psychology, School of \\ Education Science, Hunan Normal \\ University, Changsha, People's Republic \\ of China; ${ }^{2}$ Cognition and Human \\ Behavior Key Laboratory of Hunan \\ Province, Hunan Normal University, \\ Changsha, People's Republic of China; \\ ${ }^{3}$ Department of Criminal Justice, Ningxia \\ Police Vocational College, Ningxia, \\ People's Republic of China
}

Purpose: Many studies explore the relationship between moral judgment and psychopathy in western culture, but the mechanism underlying this relationship remains unclear. By far, no research about this topic in the background of Chinese culture exists. In the current study, we adopt one of the creative process-dissociation approaches to explore the relationship between the psychopath and moral judgment.

Methods: Adopt the Levenson Self-Report Psychopathic Scale, the Chinese version of Interpersonal Reactivity and Process-dissociation approach to explore the relationship between the psychopath and moral judgment.

Results: Traditional utilitarian moral score of the high psychopathy group are significantly higher than that of low psychopathy group $(t=2.97, \mathrm{p}<0.05)$, people with high psychopathy utilitarian tendency $U$ factor score and people with low psychopathy have no significant difference $(\mathrm{F}=0.85, p=0.36)$.

Conclusion: Individuals with high psychopathy tend to make fewer deontological moral judgments because of their decreased deontological tendencies rather than their increased utilitarian tendencies. They may make more acceptance choices not to increase the wellbeing of the majority of people, but because of their increased acceptance of hurting others in the moral dilemma.

Keywords: psychopathy, moral judgment, deontological inclination, utilitarian inclination

\section{Introduction}

Psychopathy is a personality disorder that includes a range of interpersonal, emotional, lifestyle, and antisocial traits as well as behavioral manifestations. Specifically, in the interpersonal aspect, they are deceptive, superficial, like to dominate, and manipulate others. In terms of emotion, they are superficial, callous, lack of sympathy, guilt, and remorse. ${ }^{1}$ These characteristics lead them to deviate from social norms by acting impulsively, irresponsibly (not necessarily criminally), ignoring or violating social rules, and even engaging in certain antisocial behaviors. $^{2}$

Cleckley classified psychopaths as primary and secondary. ${ }^{3}$ The primary psychopaths are no apparent anxiety or guilt felt after engaging in illegal or unethical behavior and guilt. The secondary psychopaths report more frequent aggressive behavior and less anxiety. Hare proposed a classification of primary, secondary, and dyssocial psychopaths and showed that psychiatric morbidity is not a personality disorder. Such morbidity appears a continuous distribution in the crowd, whereas
Correspondence: Daoqun Ding Department of Psychology, School of Education Science, Hunan Normal University, 36 Lushan Road, Changsha 4I008I, Hunan, People's Republic of China

Tel +86 I557585I428

Email psychding@hunnu.edu.cn 
the personality disorder is disorder distribution. ${ }^{4}$ Their psychiatric state is only reflected in the degree of difference. In the general population, the level of the psychopathic traits' tendency also reflects the degree of differentiation. ${ }^{5}$

Hare developed the psychopathy checklist (PCL) to evaluate psychopathy based on the descriptions of psychopathy features. He then changed PCL into a revised psychopathy checklist (PCL-R). The revised version of PCL-R in 2003 is widely used nowadays. ${ }^{6}$ However, PCL-R has certain limitations in practical operation and application. First, it has a time-consuming assessment process that requires at least 2-3 h for each assessment. Second, a part of the assessment work must be completed by consulting archives or indirect information, which is difficult to obtain in places other than prisons. Third, PCL-R studies are conducted in the clinical or incarcerated population and not in the non-incarcerated population. ${ }^{7}$

The research object extends to the general population with the deepening of researching psychopathy. Researchers found that the psychopathic traits in prison and the general population only show differences in degree. In addition, ordinary individuals show psychopathic features as the patient or the crime. ${ }^{8}$ Hence, researchers develop many selfreport scales to assess psychopathic trait tendencies in the general population. ${ }^{9}$ The self-reported scale mainly includes the following: (1) psychopathic personality inventory (PPI), ${ }^{10}$ (2) Levenson self-report psychopathy scale (LSRP), ${ }^{11,12}(3)$ Self-report psychopathy scale, and (4) Triarchic psychopathy measure. ${ }^{13}$ LSRP is among the famous scales worldwide, and numerous studies confirm the scale's good reliability and validity. LSRP has only 26 items and various samples, which include the incarcerated, the community, and the children samples. Due to its good cross-cultural characteristics, we adapt LSRP to investigate the subclinical population in the background of the Chinese culture. ${ }^{14,15}$

Moral judgment relies on affection, cognition, and complex neural circuits related to behavioral processes. Thus, it is one of the most complex activities in the field of human cognition. Many studies about moral judgment exist. ${ }^{16,17}$ Greene et al proposed a dual-process model and showed that affection and reasoning are the two independent psychological processing systems working together on moral judgment.

1. Deontological judgment is associated with an effective process. Individuals with deontological principles hold the idea that the morality of a one's behavior depends on whether the behavior is ethical or normative (eg, killing one to save five is morally wrong).

2. Utilitarian judgment is associated with the reasoning process. Individuals with utilitarian principles consider that the morality of one's behavior depends on whether the utility or outcome is maximized (eg, killing one to save five is acceptable). ${ }^{18}$

Individuals who tend to make deontological judgments in moral judgment often score high measures of affective processing. ${ }^{19,20}$ Conversely, clinical patients with affective processing defects make utilitarian judgments in moral judgments. Given the social and affective deficits of psychopaths, researchers found that psychopathy in western culture can make more utilitarian judgments. ${ }^{21-24}$ Koenigs found that primary psychopaths endorse more personal utilitarian choices in comparison with secondary psychopaths and non-psychopaths. ${ }^{25}$ Balash and Falkenbach (2018) investigated the relationship between psychopathic traits and utilitarian endorsement in a college sample and found that individuals with a high level of psychopathic traits willingly accept harmful actions in accidental harm scenarios. ${ }^{26}$ In the same year, Virgil used various sacrificial dilemmas to analyze the relationship between psychopathic traits and utilitarian moral judgments. Comparing with an impersonal dilemma, the agent needs to use personal force (ie, by executing a motor act) and involve emotion in a personal dilemma. They found that psychopathic personality traits can only predict utilitarian judgment in personal dilemmas. Hence, people with psychopathic traits can make utilitarian moral judgments for emotional defects. However, Cima and Tonnaer found no difference between the utilitarian endorsements of forensic psychopaths (indexed via PCL-R; Hare) and non-psychopaths. ${ }^{27}$ Most studies found that individuals with a high level of psychopathic traits make more utilitarian moral judgments.

Ethics also divides people's moral judgment into utilitarianism and deontologist judgment. ${ }^{28}$ Conceptually, utilitarian judgment is defined by interests that are sensitive to consequence. ${ }^{29}$ Therefore, to classify whether a given judgment is utilitarian or not, it is necessary to identify its property of being sensitive to results. Deontological judgment is defined by attributes sensitive to moral norms. Therefore, to classify whether a given judgment as 
a deontological judgment or not, its sensitivity to moral norms must be confirmed.

Utilitarianism holds that whether an act is moral depends on the effect of the result of the act on the individual's overall happiness. In contrast, deontology holds that the morality of the act depends on the consistency between the action itself and the moral norm. ${ }^{29}$ Early studies on moral judgment divided people's judgments into utilitarian or deontological tendencies and correlated these two types of judgments with controlled processing and automated processing. ${ }^{28}$ However, the theory does not take into account the possibility that subjects are motivated by both motives when making moral judgments. Recently, Gawronski et al (2013) constructed a process-dissociation approach to try to separate the different cognitive processing processes of subjects in making judgments in moral dilemmas. In this model, the researcher assumes that two psychological processes accomplish moral judgment: consequence (parameter $U$ ) and norm (parameter $D$ ). They represent the underlying mental processes of two hypotheses: utilitarian tendency and deontological tendency.

Greene and other researchers believe that affective processing and reasoning are two separate psychological systems. The affective and reasoning processing systems drive the deontological and the utilitarian inclinations, respectively. ${ }^{28-30}$ Traditional data analysis strategy implicitly or explicitly considers utilitarian and deontological inclinations to be negatively correlated. Hence, the weakening of deontological inclinations enhances utilitarianism inclinations. $^{31}$ Researches proved that individuals with a high level of psychopathy have emotional deficits. Do these individuals only have differences in deontological inclinations and no difference in utilitarian inclinations? Previous studies cannot explain if individuals with emotional deficits affect moral judgments perturbed by one of the psychological processing systems or two systems. The process-dissociation approach can separately measure the relative strength of individual deontological and utilitarian inclinations. Thus, we adopt this approach to investigate the underlying mechanism between psychopathy and moral judgment.

The process-dissociation approach uses moral materials of incongruent and congruent dilemmas.

1) In incongruent dilemmas, individuals with utilitarian or deontological principles conflict in considering harmful behavior. Individuals based on utilitarian principles tend to accept harmful behavior to maximize benefit (eg, killing one to save five people). By contrast, individuals with deontological principles consider that harmful actions violate deontological rules and tend to reject harmful behaviors. $^{29,32,33}$ For example, in the car accidents of the incongruent dilemmas, people with utilitarian principles tend to hit an older man and save a young mother and a child. By contrast, people with deontological inclinations tend to refuse to hit the older man even if they hit the young mother and the child.

2) In the congruent dilemmas, individuals based on deontological principles or utilitarian principles are compatible with considering harmful behaviors. Individuals based on the principles of deontology or utilitarianism tend to reject harm. For example, in the car accidents of congruent dilemmas, the result is described as whether to turn the steering wheel to kill a group of primary school students to save the young mother and child. Both of them tend to refuse to turn the steering wheel.

We could calculate the relative strength of deontological and utilitarian inclination for each participant by comparing choices in congruent and incongruent dilemmas. The dual-process model indicated that defects in emotional processing could affect deontological inclinations but not utilitarian inclinations. $^{29,33}$ Emotional processing defects may affect a high level of individuals' psychopathy inclinations. However, utilitarian inclinations are not affected by emotional processing. Several studies showed that expressive suppression or feeling less could selectively reduce the deontological inclinations but not utilitarian inclinations. $^{29}$ Therefore, high- and low-psychopathy individuals differ in terms of deontological inclinations. Under the background of the Chinese culture, we hypothesize that high-psychopathy individuals would make fewer deontological moral judgments in moral dilemmas than low-psychopathy individuals. This assumption is not affected by the increase of utilitarian inclinations but by the decrease of deontological inclinations.

\section{Method}

\section{Participants}

We recruited 167 participants aged over 18, comprising 83 males and 84 females. Those aged between 18 and 25 accounted for $82.24 \%$ of the total number of participants. All participants were right-handed, with a normal or corrected-to-normal vision-all participants involved in the present study signed informed consent. The research protocol was approved by the ethics committee. The 
research was conducted in accordance with the Declaration of Helsinki. The first $20 \%$ of participants are in the group with high-psychopathy individuals, according to LSRP. The last $20 \%$ is in the group with low-psychopathy individuals. A total of 33 participants were gathered $(M=92.55, S D=5.88)$ in the people with high psychopathy, including 24 men.Moreover, 33 participants were in the people with low psychopathy $(M=$ 53.06, $S D=7.45$ ), including 16 men. A significant difference is observed in psychopathy trait scores between the two groups, $\mathrm{t}=17.23, \mathrm{p}=0.00$. In the people with high psychopathy, men significantly outnumber women, $\chi^{2}(1, \mathrm{~N}=33)=6.82, d f=1, \mathrm{p}=0.01$.

\section{Materials}

\section{The Chinese Version of the Levenson Self-Report Psychopathy Scale (LSRP)}

We use a validated Chinese version of the Levenson SelfReport Psychopathy Scale (LSRP). ${ }^{12}$ Items are rated using a 6-point Likert scale; each is coded from 1 (strongly disagree) to 5 (strongly agree). Higher total score indicates a higher level of psychopathy trait. The Cronbach's $\alpha$ coefficient for the LSRP was 0.85 in the present research.

\section{The Interpersonal Reactivity Index}

We use validated the Chinese version of the Interpersonal Reactivity Index, ${ }^{34}$ it is rated using a 5-point Likert scale, each coded from 1 (strongly disagree) to 5 (strongly agree). Higher total score indicates a higher level of empathy. The Cronbach's $\alpha$ coefficient for the scale is 0.76 in the present research.

\section{Experimental Task and Procedure}

We adapt the process-dissociation task, which included ten incongruent and ten congruent moral dilemmas. These dilemmas are designed to provide separate measures of utilitarian and deontological inclinations for each participant. $^{29,31}$ In incongruent dilemmas, the participant's utilitarian and deontological inclinations lead to different responses. For instance, in the incongruent dilemma of a car accident, we ask participants whether swerving and hitting an old lady to avoid hurting a young mother and her child is acceptable. In this case, utilitarian inclinations lead people to accept hitting the old lady, whereas deontological inclinations lead people to reject hitting the old lady.
However, incongruent dilemmas, utilitarian, and deontological inclinations will lead to the same responses. For instance, in the congruent dilemma of a car accident, we ask participants whether swerving and hitting a group of schoolchildren to avoid hurting a young mother and her child is acceptable. In this case, utilitarian and deontological inclinations lead people to reject hitting the group of schoolchildren. Comparing the response rates for incongruent and congruent dilemmas allowed us to quantify the strength of utilitarian and deontological inclinations independently.

Accordingly, we follow the procedure detailed in Conway and Gawronski (2013). A utilitarianism ( $U$ ) parameter is calculated by taking the difference in the proportion of "refuse" responses between the congruent and incongruent dilemmas: $U=p$ (unacceptable/congruent) $p$ (unacceptable/incongruent). The score of $U$ ranges from -1 to 1 .

The high score of $U$ indicates that participants tend to accept harmful actions when they maximized the net benefit (ie, incongruent dilemmas). Otherwise, they refuse such actions (ie, congruent dilemmas).

A deontology $(D)$ parameter is calculated as the proportion of "unacceptable" responses in incongruent dilemmas relative to all non-utilitarian responses:

$$
D=p(\text { unacceptable/incongruent }) /(1-\mathrm{U})
$$

The score of $D$ ranges from 0 to 1 . The high score of $D$ indicates that the participants tend to reject harmful actions, though they can maximize the net benefit.

In addition, a traditional $(T)$ bipolar score of utilitarianism versus deontology is derived by calculating the proportion of "acceptable" responses in incongruent dilemmas:

$$
T=p(\text { acceptable/incongruent })
$$

The score of $T$ ranges from 0 to 1 . High score of $T$ indicates many utilitarian judgments and few deontological judgments.

This experiment has one block, which comprises 20 trials (ten incongruent and ten congruent dilemma trials), and these trails are in a pseudorandom order. Each trial begins with a central fixation point, and the duration of each trial varies from 600 to $1000 \mathrm{~ms}$. Subsequently, a moral dilemma question and two choices are simultaneously presented on the screen. The participants are asked to indicate whether the described action is acceptable or not. Following their personal opinion, these 


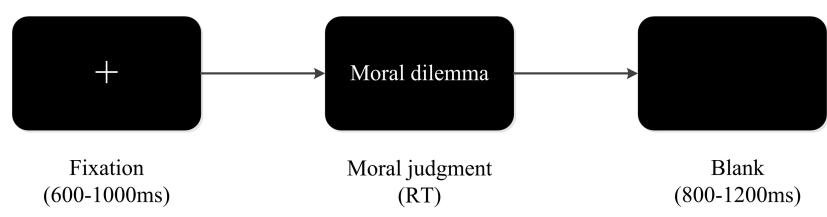

Figure I Illustration of the experimental procedure. At the moral judgment phase, incongruent and congruent dilemma trials present in a pseudo-random order.

participants press " 1 " to select "acceptable" and " 0 " to select "refuse". These moral and alternatives dilemmas remained on the screen until the participants make a choice. Following their responses, each trial ends with a blank screen that continues 800 to $1200 \mathrm{~ms}$ (see Figure 1).

\section{Results}

Table 1 presents the descriptive statistics and correlations between the nonstandard variables. The traditional bipolar score is positively correlated with $U, r=0.57, \mathrm{p}<0.001$ and negatively correlated with $D, r=-0.72, \mathrm{p}<0.001$. However, the two parameters are statistically independent of each other, $r=0.13, \mathrm{p}=0.09$. Moreover, $D$ negatively correlates with psychopathy, $r=0.36, \mathrm{p}<0.01$, whereas $U$ negatively correlates with empathy $r=-0.21, \mathrm{p}<0.01$. A significant negative correlation is observed between empathy and psychopathy $r=-0.21, \mathrm{p}<0.01$. These results (see Supplementary data) prove the validity of the process dissociation approach and provide preliminary support for further testing of hypotheses.

\section{Traditional Analysis}

Traditional bipolar score is calculated with the T equation. Results reveal that the participants with high psychopathy $(M=0.40, S D=0.22)$ show a stronger preference for utilitarian judgments more deontological judgments than those with low psychopathy $(\mathrm{M}=0.25, \mathrm{SD}=0.18), \mathrm{t}(33)$ $=2.97, \mathrm{p}<0.05$, Cohen's $d=0.83$ (see Figure 2).

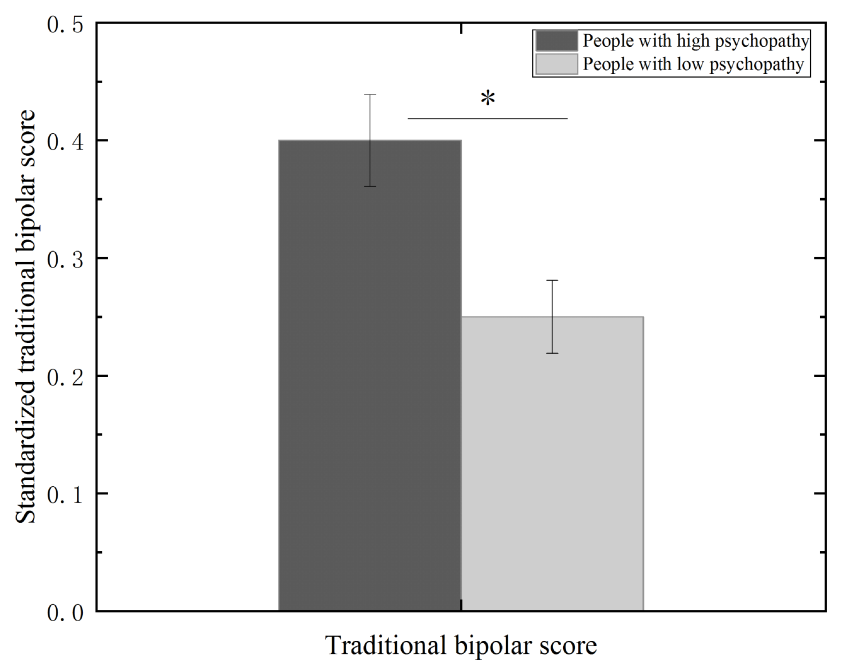

Figure 2 The traditional bipolar score for people with high and low psychopathy. Error bars indicate standard errors. ${ }^{*} p<0.05$

\section{Process-Dissociation Analysis}

The two parameter scores are standardized to investigate the effect of psychopathy on moral judgment. A 2 (group: people with high psychopathy vs people with low psychopathy) $\times 2$ (parameter: deontology vs utilitarianism) repeats measures analysis of variance is then conducted with a group as a between-subject variable and process dissociation (PD) parameter as a within-subject variable. The analysis reveals that the interaction effect between the group and PD parameter is significant $F(1,66)=6.23, \mathrm{p}=0.018, \eta_{\mathrm{p}}{ }^{2}$ $=0.16$. Follow-up simple effects analysis reveals that deontological inclinations (ie deontology parameter) are significantly lower in the people with high psychopathy $(\mathrm{M}=0.51$, $\mathrm{SD}=0.16)$ than that in the people with low psychopathy ( $\mathrm{M}$ $=0.63, \mathrm{SD}=0.14), F(1,66)=9.11, \mathrm{p}=0.01, \eta_{\mathrm{p}}{ }^{2}=0.22$. However, the difference of utilitarian inclinations (ie utilitarian parameter) between the people with high psychopathy $(M=-0.16, S D=0.23)$ and people with low psychopathy $(\mathrm{M}=-0.20, \mathrm{SD}=0.21)$ is non-significant, $F(1,66)=0.85$, $\mathrm{p}=0.36, \eta_{\mathrm{p}}{ }^{2}=0.03$. (see Figure 3 ).

Table I Descriptive Statistics and Correlations Between All Variables ( $\mathrm{N}=167)$

\begin{tabular}{|l|l|l|l|l|l|l|}
\hline Variable & $\mathbf{M}$ & SD & I & $\mathbf{2}$ & $\mathbf{3}$ & $\mathbf{4}$ \\
\hline I Traditional bipolar score (T) & 0.30 & 0.19 & - & & \\
2 PD utilitarianism (U) & -0.19 & 0.22 & $0.57^{* *}$ & - & \\
3 PD deontology (D) & 0.59 & 0.15 & $-0.72^{* *}$ & 0.13 & - & \\
4 Psychopathy & 71.74 & 14.14 & $0.36^{* *}$ & 0.10 & $-0.35^{* *}$ \\
5 empathy & 46.25 & 9.68 & -0.10 & $-0.21^{* *}$ & -0.03 & - \\
\hline
\end{tabular}

Note: $* * p<0.01$.

Abbreviation: PD, process dissociation. 


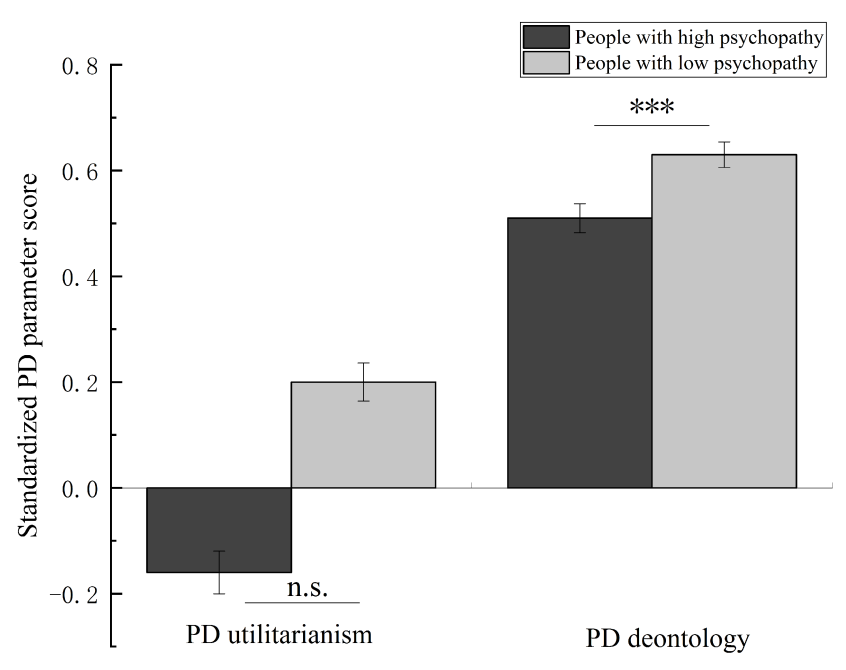

Figure 3 Mean standardized PD deontology and utilitarianism score for the people with high and low psychopathy. Error bars indicate standard errors. $* * * p<0.001$, n.s. indicates non-significant differences as $p>0.05$.

\section{Regression Analysis}

We use multiple regression to analyze unique relations of the Process Dissociation further, and PD deontology and PD utilitarianism estimate to the employed variables in Chinese samples. These analyses were exploratory, as limited research analyzed the differential contributions of Psychopathy in the context of Process Dissociation, we simultaneously regressed each variable onto both the PD deontology and utilitarianism parameters (see Table 2). Corroborating the results of our correlation analyses, utilitarianism, but not deontology, emerged as a significant predictor of empathy, whereas, deontology, but not utilitarianism, showed a significant relation to Psychopathy. However, both Deontology inclinations and Utilitarianism inclinations independently predicted a traditional bipolar score.

\section{Mediation Analysis}

We analyze whether PD deontological inclinations mediated the relationships between psychopathy and

Table 2 Multiple Regression Analyses Regressing Theoretically Relevant Variables Onto PD Deontology and PD Utilitarianism Scores

\begin{tabular}{|l|l|l|l|l|l|l|}
\hline \multirow{2}{*}{ Variable } & \multicolumn{3}{|l|}{ PD Deontology } & \multicolumn{3}{l|}{ PD Utilitarianism } \\
\cline { 2 - 7 } & $\boldsymbol{\beta}$ & $\boldsymbol{t}$ & $\boldsymbol{P}$ & $\boldsymbol{\beta}$ & $\boldsymbol{t}$ & $\boldsymbol{P}$ \\
\hline $\begin{array}{l}\text { Traditional } \\
\text { bipolar score }\end{array}$ & -0.72 & -13.33 & $<0.01$ & 0.57 & 8.94 & $<0.01$ \\
Psychopathy & -0.35 & -4.84 & $<0.01$ & 0.10 & 1.33 & 0.18 \\
Empathy & -0.03 & -0.42 & 0.68 & -0.21 & -2.72 & $<0.01$ \\
\hline
\end{tabular}

Abbreviation: $\mathrm{PD}$, process dissociation.

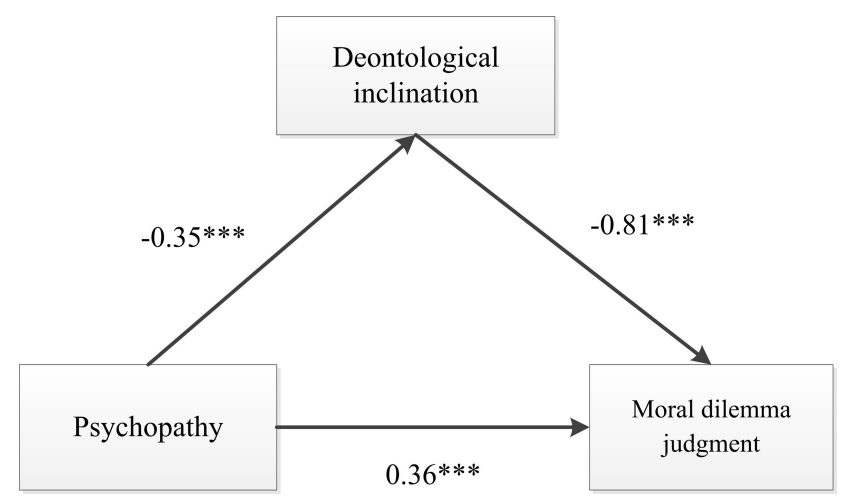

Figure 4 Deontological inclination mediated the relationship between psychopathy and moral dilemma judgment. The standardized coefficients when the mediator is included in the model are presented above the arrow. ${ }^{* * *} p<0.001$.

moral dilemma judgment. The bootstrap procedure with 5000 resamples is adopted to investigate the significance levels of indirect effects. Results reveal that deontological tendency plays an intermediary role between groups and moral judgments. The indirect effect of psychopathy on moral dilemma judgment via PD deontological inclinations is significant $\beta=0.69, \mathrm{SE}=0.08,95 \% \quad \mathrm{CI}=$ $[0.53,0.86]$. Moreover, psychopathy negatively affects deontological inclinations, which in turn significantly and negatively affected moral dilemma judgment $(\beta=-0.81$, $p<0.001)$. Besides, we control the deontological tendency of the intermediary variable. However, the independent variable psychopathy still did significantly influence the traditional moral judgment of the dependent variable $\beta=$ $0.002, \mathrm{SE}=0.001,95 \% \mathrm{CI}=[0.0001,0.003]$. Figure 4 shows that the deontological tendency plays a partially mediating role in moral judgment between psychopathy and moral dilemma judgment.

\section{Discussion}

In the present study, the process dissociation paradigm is employed to investigate the moral judgment characteristics among the psychopath population and to discuss its internal mechanism. Firstly, such a paradigm verified that individuals with high psychopathy tend to make fewer deontological moral judgments in a moral dilemma. Secondly, the deontological tendency score of people with high psychopathy is significantly lower than the score of people with low psychopathy. However, utilitarianism tendency of both groups is not significantly different. The reason why individuals with high psychopathy make a fewer deontological judgment is revealed. Based on previous findings, the utilitarian moral judgment made by individuals in a dilemma is driven by an 
antisocial tendency to a certain degree. ${ }^{35-37}$ The reason why psychopaths tend to make fewer deontological moral judgments may be that they lack concerns for individuals to be damaged but not for additional benefits. Individuals with high psychopathy may make decisions that can increase the benefits of a broad population. However, they act as such probably because they do not care about those who are going to be damaged. Pletti et al (2016) have a similar view. Psychopaths tend to make fewer deontological judgments in a sacrificial dilemma only because they consider that emotional disturbance decreases when hurting another individual. When psychopaths make a moral judgment, they lack concerns for others' injuries, and understanding others' psychological states is difficult for them. ${ }^{38}$ In the end, the mediation analysis shows that the deontological tendency mediates the relationship between psychopathy and moral judgment. These findings are consistent with our hypothesis and the dual process model. ${ }^{39}$

Firstly, the present study verified in Chinese cultural background that individuals with high psychopathy are likely to perform fewer deontological moral judgments. Besides, conventional deontological judgments are significantly lower in the high psychopathy group than in the low-psychopathy group. Individuals with high psychopathy likely accept injurious behavior in moral judgment. This result is consistent with the findings of Justin Balasha et al. ${ }^{26,40}$ But different from those of Cima. This difference may be because the technique adopted in the present study is LSRP, which is suitable for ordinary subjects. Also, the process dissociation paradigm used in the present study is different from the traditional moral dilemma. Current studies about the relation between psychopathy and moral judgment are all from foreign countries. The present study firstly explored and then verified the relationship in Chinese culture.

Previous studies do not measure the deontological and utilitarian tendencies separately. Therefore, whether the emotional defects of psychopaths will influence their moral judgment by affecting the two processing systems or either one of them cannot be determined. ${ }^{41,42}$ The present study effectively solves this problem by using the process dissociation technique. The score in deontological tendency is significantly lower in people with high psychopathy than that in people with low psychopathy. Moreover, utilitarianism tendency is not significantly different between the two groups. Results indicate that the emotional defects of psychopaths affect their emotional processing system. Several studies strongly support the current findings. Physical arousal is low in moral decisions among individuals with high psychopathy. Hence, this population experiences a few mental conflicts in an ethical dilemma. Studies show that the rise in characteristics of psychopathy is related to the increased risk of instrumental aggression. This relation is affected by the reaction of the amygdala to pain signals. ${ }^{43}$ In short, a weak reaction ability to others' pain is correlated to a reduced sense of guilt and empathy and a rise in instrumental aggression. Functional magnetic resonance imaging research indicates that the amygdala is one of the core regions for moral judgment and is always in an active state in moral judgment tasks. However, the role of the amygdala in moral judgment is damaged among individuals with psychopathy. By contrast, moral judgment does not have much correlation with the activation of the amygdala. ${ }^{44}$

In contrast to our prediction, we failed to find empathy to predict actual moral judgment. Results indicate that all those people who score high on psychopathy make fewer deontological judgments for the emotional and behavior deficit regardless of empathy level. The present study finds that empathy is significantly and negatively correlated to psychopathy and $U$ score. However, empathy cannot directly influence the utilitarian moral judgment. This result is not consistent with the previous research. ${ }^{45}$ One possible reason is that the previous study investigated the association in the western culture samples, whereas we focused on the Chinese non-institutionalized college samples. The difference in the screening criteria of subjects may be the primary reason for the inconsistency of the present study with previous research. In the study of Ana Seara-Cardoso, ${ }^{46}$ all participants were women, whereas the present study included male subjects.

According to Hare, the morbidity of psychopathy among healthy individuals is approximately $1 \%-3 \%$, and this rate among adult criminals is approximately $15 \%-30 \%$. Psychopathy is a kind of personality disorder closely related to antisocial behaviors such as attack and violencel. Criminals with the trend of psychopathy have a high recidivism rate, specifically for violent crimes. Considering the severity of this disease, researchers and clinical physicians should get to know the moral cognitive characteristics and their development rules among the psychopath population. This study found that the moral dilemma judgment of psychopaths is influenced by the deontological tendency that mostly involves emotional processing. In the future, the intervention should focus on cultivating the focus and experience of individuals with high psychopathy on negative emotions, precisely the moral sentiment of children and teenagers with Callous-Unemotional (CU) traits. Immoral behavior can be effectively inhibited by promoting the benign 
development of their moral cognition, thus providing a specific theoretical basis for the psychotherapy of psychopaths. Certain studies found that individuals with emotional deficits or clinical patients likely perform utilitarian judgment. However, its potential mental mechanism is not precise. Therefore, the process dissociation paradigm can be adopted in further research to explore the possible psychological mechanism of moral judgment among these individuals.

\section{Conclusions}

The current results contribute to the relationship between moral judgments and psychopathy in Chinese culture. The study finds that individuals with high psychopathy tend to make a fewer deontological judgments in moral dilemmas. The deontological inclinations of individuals with high psychopathy can affect their moral judgments. Besides, the moral decision of those individuals does not influence by their utilitarian inclinations.

The present study provides new insight into the followup research. First, the measurement method in the present study is for self-report. However, such a method is not the comprehensive measurement for physiological indexes, including electroencephalography, skin conductance, and heart rate. In the future, physiological indexes can be combined to record the moral judgment characteristics of psychopaths. Secondly, college students are chosen as the object of the current study, which did not include criminals and clinical populations. Hence, the sample size can be expanded in the follow-up studies. Finally, the difference and similarity in moral judgment characteristics between CU featured children and teenagers and adult psychopath populations can be checked longitudinally in succeeding research. Such a process can deepen the understanding of the moral development of psychopathy and the specific pathological personality trait.

\section{Ethical Approval}

The Institutional Review Board (IRB) of the Hunan Normal University in Hunan approved the study (2019224).

\section{Data Sharing Statement}

The data described in this article are openly available in the Open Science Framework at DOI:10.17605/OSF.IO/TPA6U.

\section{Acknowledgments}

This work was supported by the Social Science Foundation of Hunan Province, China (17YBA278) and the Provincial Innovation Foundation For Postgraduate (CX20190377).

\section{Funding}

Supported by the Social Science Foundation of Hunan Province, China (17YBA278) and the Hunan Provincial Innovation Foundation For Postgraduate (CX20190377).

\section{Disclosure}

The authors have declared no competing interests.

\section{References}

1. Hare RD, Neumann CS. Psychopathy as a clinical and empirical construct. Annu Rev Clin Psychol. 2008;4(1):217-246. doi:10.1146/ annurev.clinpsy.3.022806.091452

2. Blair RJ. Emotion-based learning systems and the development of morality. Cognition. 2017;167:38-45. doi:10.1016/j.cognition.2017. 03.013

3. Cleckley, Hervey. Personality and the behavior disorders. $J$ Nerv Ment Dis. 1945;102(1):93-94. doi:10.1097/00005053-19450700000024

4. Hare, Robert D. A research scale for the assessment of psychopathy in criminal populations. Pers Individ Dif. 1980;1(2):111-119. doi:10.1016/0191-8869(80)90028-8

5. Ishikawa SS, Raine A, Lencz T, Bihrle S, Lacasse L. Autonomic stress reactivity and executive functions in successful and unsuccessful criminal psychopaths from the community. J Abnorm Psychol. 2001;110(3):423-432. doi:10.1037/0021-843X.110.3.423

6. Hare RD. The Hare Psychopathy Checklist-Revised. 2nd ed. Toronto: Multi-Health Systems; 2003.

7. Neal TMS, Sellbom M. Examining the factor structure of the hare self-report psychopathy scale. J Pers Assess. 2012;94(3):244-253. doi:10.1080/00223891.2011.648294

8. Hare RD. Comparison of procedures for the assessment of psychopathy. J Consulting Clin Psychol. 1985;53(1):7. doi:10.1037/ 0022-006X.53.1.7

9. Lilienfeld SO, Andrews BP. Development and preliminary validation of a self-report measure of psychopathic personality traits in noncriminal population. $J$ Pers Assess. 1996;66(3):488-524. doi:10.1207/s15327752jpa6603_3

10. Brinkley CA, Schmitt WA, Smith SS, Newman JP. Construct validation of a self-report psychopathy scale: does levenson's self-report psychopathy scale measure the same constructs as hare's psychopathy checklist-revised? Pers Individ Dif. 2001;31(7):1021-1038. doi:10.1016/S0191-8869(00)00178-1

11. Christian E, Sellbom M. Development and validation of an expanded version of the three-factor levenson self-report psychopathy scale. J Pers Assess. 2015;98(2):1-14. doi:10.1080/00223891.2015.1107728

12. Somma A, Fossati A, Patrick C, Maffei C, Borroni S. The threefactor structure of the levenson self-report psychopathy scale: fool $/ \mathrm{s}$ gold or true gold? A study in a sample of italian adult non-clinical participants. Personal Ment Health. 2014;8(4):337-347. doi:10.1002/ pmh. 1267

13. Shou Y, Sellbom M, Xu J, Chen T, Sui A. Elaborating on the construct validity of triarchic psychopathy measure in chinese clinical and nonclinical samples. Psychol Assess. 2017;29:1071. 
14. Shou Y, Sellbom M, Han J. Evaluating the construct validity of the levenson self-report psychopathy scale in China. Assessment. 2016;24:1008-1023.

15. Sellbom M. Elaborating on the construct validity of the levenson self-report psychopathy scale in incarcerated and non-incarcerated samples. Law Human Behav. 2011;35(6):440-451. doi:10.1007/ s10979-010-9249-x

16. Gleichgerrcht E, Tomashitis B, Sinay V. The relationship between alexithymia, empathy and moral judgment in patients with multiple sclerosis. Eur J Neurol. 2015;22(9):1295-1303. doi:10.1111/ ene.2015.22.issue-9

17. Greene JD. An fmri investigation of emotional engagement in moral judgment. Science. 2001;293(5537):2105-2108. doi:10.1126/ science. 1062872

18. Greene J, Haidt J. How (and where) does moral judgment work? Trends Cogn Sci. 2003;6(12):517-523. doi:10.1016/S1364-6613(02) 02011-9

19. Greene JD. The rise of moral cognition. Cognition. 2015;135(Sp. Iss. SI):39-42. doi:10.1016/j.cognition.2014.11.018

20. Greene JD. Emotion and morality: a tasting menu. Emotion Rev. 2011;3(3):227-229. doi:10.1177/1754073911409629

21. Patil I. Trait psychopathy and utilitarian moral judgment: the mediating role of action aversion. J Cognit Psychol. 2015;27(3):349-366. doi:10.1080/20445911.2015.1004334

22. Reynolds CJ, Conway P. Not just bad actions: affective concern for bad outcomes contributes to moral condemnation of harm in moral dilemmas. Emotion. 2018;18(7):1009-1023. doi:10.1037/emo0000 413

23. Gao Y, Tang S. Psychopathic personality and utilitarian moral judgment in college students. J Crim Justice. 2013;41(5):342-349. doi:10.1016/j.jcrimjus.2013.06.012

24. Vyas K, Jameel L, Bellesi G, Crawford S, Channon S. Derailing the trolley: everyday utilitarian judgments in groups high versus low in psychopathic traits or autistic traits. Psychiatry Res. 2017;250 (Complete):84-91. doi:10.1016/j.psychres.2017.01.054

25. Koenigs M, Kruepke M, Zeier J, Newman JP. Utilitarian moral judgment in psychopathy. Social Cognit Affective Neurosci. 2012;7 (6):708-714. doi:10.1093/scan/nsr048

26. Balash J, Falkenbach DM. The ends justify the meanness: an investigation of psychopathic traits and utilitarian moral endorsement Pers Individ Dif. 2018;127:127-132. doi:10.1016/j.paid.2018.02.009

27. Cima M, Tonnaer F, Hauser MD. Psychopaths know right from wrong but don\"t care. Soc Cogn Affect Neurosci. 2010;5(1):59-67. doi:10.1093/scan/nsp051

28. Greene JD. Why are VMPFC patients more utilitarian? A dual-process theory of moral judgment explains. Trends Cogn Sci. 2007;11(8):322-323. doi:10.1016/j.tics.2007.06.004

29. Conway P, Gawronski B. Deontological and utilitarian inclinations in moral decision making: a process dissociation approach. J Pers Soc Psychol. 2013;104(2):216. doi:10.1037/a0031021

30. Hayakawa S, Tannenbaum D, Costa A, Corey JD, Keysar B. Thinking more or feeling less? Explaining the foreign-language effect on moral judgment. Psychol Sci. 2017;28(10):1387-1397. doi:10.1177/0956797617720944
31. Li Z, Xia S, Wu X, Chen Z. Analytical thinking style leads to more utilitarian moral judgments: an exploration with a process-dissociation approach. Pers Individ Dif. 2018;131:180-184. doi:10.1016/j.paid.2018.04.046

32. Greene JD, Morelli SA, Lowenberg K, Nystrom LE, Cohen JD. Cognitive load selectively interferes with utilitarian moral judgment. Cognition. 2008;107(3):1144-1154. doi:10.1016/j. cognition.2007.11.004

33. Lee JJ, Gino F. Poker-faced morality: concealing emotions leads to utilitarian decision making. Organ Behav Hum Decis Process. 2015;126:49-64. doi:10.1016/j.obhdp.2014.10.006

34. Davis MH. Measuring individual differences in empathy: evidence for a multidimensional approach. J Pers Soc Psychol. 1983;44 (1):113-126. doi:10.1037/0022-3514.44.1.113

35. Yilmaz O, Harma M, Bah Ekapili HG, Cesur S. Validation of the moral foundations' questionnaire in turkey and its relation to cultural schemas of individualism and collectivism. Pers Individ Dif. 2016;99:149-154. doi:10.1016/j.paid.2016.04.090

36. Kahane G, Everett JAC, Earp BD, Farias M, Savulescu J. 'Utilitarian' judgments in sacrificial moral dilemmas do not reflect impartial concern for the greater good. Cognition. 2015;134:193-209. doi:10.1016/j.cognition.2014.10.005

37. Pletti C, Lotto L, Buodo G, Sarlo M. It's immoral, but I'd do it! Psychopathy traits affect decision-making in sacrificial dilemmas and in everyday moral situations. Br J Psychol. 2016;108:351-368.

38. Viding E, Mccrory EJ. Understanding the development of psychopathy: progress and challenges. Psychol Med. 2017;48(4):1.

39. Greene JD. The rat-a-logical imperative: moral intuition and the limits of effective learning. Cognition. 2017;167:66-77.

40. Seara-Cardoso A, Neumann C, Roiser J, Mccrory E, Viding E. Investigating associations between empathy, morality and psychopathic personality traits in the general population. Personality Individual Differences. 2012;52(1):67-71. doi:10.1016/j. paid.2011.08.029

41. Boccia M, Piccardi L, Guariglia P. How treatment affects the brain: meta-analysis evidence of neural substrates underpinning drug therapy and psychotherapy in major depression. Brain Imaging Behav. 2016;10(2):619-627. doi:10.1007/s11682-015-9429-x

42. Yoder KJ, Harenski C, Kiehl KA, Decety J. Neural networks underlying implicit and explicit moral evaluations in psychopathy. Transl Psychiatry. 2015;5(8):e625. doi:10.1038/tp.2015.117

43. White SF, Zhao H, Leong KK, Smetana JG, Nucci LP, Blair RJR. Neural correlates of conventional and harm/welfare-based moral decision-making. Cognit Affective Behav Neurosci. 2017;17:1114-1128. doi:10.3758/s13415-017-0536-6

44. Friesdorf R, Conway P, Gawronski B. Gender differences in responses to moral dilemmas: a process dissociation analysis. Personality Social Psychol Bulletin. 2015;41(5):696-713. doi:10.1177/0146167215575731

45. Reynolds CJ, Conway P. Not just bad actions: affective concern for bad outcomes contributes to moral condemnation of harm in moral dilemmas. Emotion. 2018;18:1009-1023. doi:10.1037/emo0000413

46. Seara-Cardoso A, Dolberg H, Neumann C, Roiser JP, Viding E. Empathy, morality and psychopathic traits in women. Pers Individ Dif. 2013;55(3):328-333. doi:10.1016/j.paid.2013.03.011 


\section{Publish your work in this journal}

Psychology Research and Behavior Management is an international, peer-reviewed, open access journal focusing on the science of psychology and its application in behavior management to develop improved outcomes in the clinical, educational, sports and business arenas. Specific topics covered in the journal include: Neuroscience, memory and decision making; Behavior modification and management; Clinical applications; Business and sports performance management; Social and developmental studies; Animal studies. The manuscript management system is completely online and includes a very quick and fair peer-review system, which is all easy to use. Visit http://www. dovepress.com/testimonials.php to read real quotes from published authors. 